\title{
FATORES ASSOCIADOS A LESÕES DE PELE DECORRENTES DO PERÍODO INTRAOPERATÓRIO
}

\author{
Factors associated with skin lesions resulting during the intraoperative period
}

\author{
Factores asociados a lesiones de piel resultantes del periodo intraoperatorio
}

\author{
Mayara Beatriz Gonçalo Bezerra' (D), Mayana Camila Barbosa Galvão² (D), José Cristovam Martins Vieira ${ }^{3}$ (D), \\ Marcella Gomes dos Santos Lopes ${ }^{4}$ (D), Aracele Tenório de Almeida e Cavalcanti ${ }^{5}$ (D), Eduardo Tavares Gomes ${ }^{6}$
}

RESUMO: Objetivo: Avaliar a ocorrência de lesões de pele no período intraoperatório decorrentes de procedimentos cirúrgicos realizados em um hospital universitário de grande porte. Método: Estudo transversal, descritivo, com abordagem quantitativa, realizado em um hospital universitário do Recife, Pernambuco, Brasil. A amostra foi constituída de 154 pacientes submetidos a cirurgias eletivas entre os meses de janeiro e abril de 2018, avaliados nos períodos pré e pós-operatório, por meio da aplicação da Escala de Avaliação de Risco para o Desenvolvimento de Lesões Decorrentes do Posicionamento Cirúrgico (ELPO), no intraoperatório. A coleta de dados ocorreu na visita pré-operatória e no primeiro dia de pós-operatório. Resultados: Dos 154 pacientes avaliados, sete apresentaram 11 lesões de pele, como lesões por pressão estágio I, estágio II e abrasão, predominantemente no glúteo e no tórax. As variáveis tempo do procedimento cirúrgico, tipo de anestesia e comorbidades foram os principais fatores de associados à ocorrência das lesões. Conclusão: Entre os pacientes que apresentaram lesões de pele devido ao posicionamento cirúrgico, a maioria foi classificada pela ELPO como alto risco, o que leva a crer que se trata de uma escala adequada para avaliação de risco de lesões de pele em pacientes cirúrgicos.

Palavras-chave: Enfermagem perioperatória. Pele. Lesão por pressão. Procedimentos cirúrgicos operatórios.

ABSTRACT: Objective: To evaluate the occurrence of skin lesions in the intraoperative period due to surgical procedures performed at a large university hospital. Method: A cross-sectional, descriptive study with a quantitative approach, performed at a university hospital in Recife, Pernambuco, Brazil. The sample consisted of 154 patients undergoing elective surgeries between January and April 2018. They were evaluated in the pre and postoperative periods, through the application of the Risk Assessment Scale for the Development of Injuries due to Surgical Positioning (ELPO), during the intraoperative period. Data were collected at the preoperative visit and on the first postoperative day. Results: Of the 154 patients evaluated, seven presented 11 skin lesions, such as stage I, stage II and abrasion lesions, predominantly in the gluteus and thorax. The variables length of the surgical procedure, type of anesthesia and comorbidities were the main factors associated with the occurrence of lesions. Conclusion: Among patients who presented skin lesions due to surgical positioning, most were classified by the ELPO as high risk, which suggests that this is a suitable scale for assessing the risk of skin lesions in surgical patients.

Keywords: Perioperative nursing. Skin. Pressure ulcer. Operative surgical procedures.

RESUMEN: Objetivo: Evaluar la ocurrencia de lesiones de piel en el período intraoperatorio resultantes de procedimientos quirúrgicos realizados en un hospital universitario de gran porte. Método: Estudio transversal, descriptivo, con abordaje cuantitativo, realizado en un hospital universitario de Recife, Pernambuco, Brasil. La muestra fue constituida de 154 pacientes sometidos a cirugías electivas entre los meses de enero y abril de 2018 , valuados en los períodos pre y postoperatorio, por medio de la aplicación de la Escala de Evaluación de Riesgo para el Desarrollo de Lesiones Debidas al Posicionamiento Quirúrgico (ELPO), en el intraoperatorio. La recolección de datos ocurrió en la visita preoperatoria y en el primer día de postoperatorio. Resultados: De los 154 pacientes evaluados, siete presentaron 11 lesiones de piel, como lesiones por presión estadio I, II y abrasión, predominantemente en el glúteo y en el tórax. Las variables tiempo del procedimiento quirúrgico, tipo de anestesia y comorbilidades fueron los principales factores de asociados a la ocurrencia de las lesiones. Conclusión: Entre los pacientes que representaron lesiones de piel debido al posicionamiento quirúrgico, la mayoría fue clasificada por ELPO como alto riesgo, lo que lleva a creer que se trata de una escala adecuada para la evaluación del riesgo de lesiones de piel en pacientes quirúrgicos. Palablas clave: Enfermería perioperatoria. Piel. Úlcera por presión. Procedimientos quirúrgicos operativos.

'Enfermeira; especialista em Enfermagem Cirúrgica pelo Programa de Residência em Enfermagem do Hospital das Clínicas da Universidade Federal de Pernambuco (HC/UFPE) - Recife (PE), Brasil. 2Enfermeira; mestre em Enfermagem pela UFRN. Preceptora do Programa de Residência em Enfermagem do HC/UFPE - Recife (PE), Brasil.

${ }^{3}$ Enfermeiro estomaterapeuta pela Universidade de São Paulo (USP); mestre em Enfermagem pela Universidade Federal de Santa Catarina (UFSC). Professor do Departamento de Enfermagem da UFPE - Recife (PE), Brasil. 'Enfermeira; mestre em Hebiatria pela Universidade de Pernambuco (UPE); especialista em Enfermagem Cirúrgica. Preceptora do Programa de Residência em Enfermagem do HC/UFPE - Recife (PE), Brasil. ${ }^{5}$ Enfermeira; mestre e doutoranda em Ciências da Saúde pela UPE. Preceptora do Programa de Residência em Enfermagem do Hospital das Clínicas da HC/UFPE - Recife (PE), Brasil.

${ }^{6}$ Doutorando em Ciências pelo Programa de Pós-Graduação em Enfermagem em Saúde do Adulto da Escola de Enfermagem da USP. Preceptor do Programa de Residência em Enfermagem do HC/UFPE-Recife (PE), Brasil.

*Autor correspondente: edutgs@hotmail.com

Recebido: 18/07/2018 - Aprovado: 04/05/2019

DOI: $10.5327 / Z 1414-4425201900020005$ 


\section{INTRODUÇÃo}

O ambiente cirúrgico é considerado de risco para iatrogenias, em razão das próprias características do cuidar, da diversidade dos procedimentos cirúrgicos e de diagnósticos, bem como da intensa circulação de profissionais, compondo uma das unidades do hospital em que os eventos adversos (EA) são mais frequentes, sendo evitáveis em até $43 \%$ dos $\operatorname{casos}^{1,2}$.

Os EA são danos ou lesões originárias do cuidado que resultam em incapacidade ou disfunção, temporária ou permanente, e até mesmo em morte entre os usuários dos serviços de saúde ${ }^{3}$.

Entre os EA que ocorrem no período perioperatório, destacam-se as complicações decorrentes do posicionamento cirúrgico: dor musculoesquelética, deslocamento de articulações, danos em nervos periféricos, lesões de pele (LP), comprometimento cardiovascular e pulmonar e síndrome compartimental ${ }^{4}$. As LP ocasionadas no período intraoperatório podem variar desde eritema que comprometa a integridade da pele a lesões extensas como queimaduras causadas por bisturi elétrico e acessórios, ou mesmo lesões por fricção ou cisalhamento, em decorrência da exposição cirúrgica sem os devidos cuidados de prevenção no posicionamento. Independentemente da posição do paciente, as proeminências ósseas e áreas de alta pressão devem ser protegidas 5 .

Estudos recentes destacaram vários fatores de risco associados a LP em pacientes cirúrgicos, e tais fatores são divididos em dois grupos: intrínsecos, como idade, peso corporal, estado nutricional e doenças crônicas como diabetes mellitus, vasculopatias, neuropatias, hipertensão e anemia; e extrínsecos, por exemplo, tipo e tempo de cirurgia, anestesia, posições cirúrgicas e posicionamento. A intensidade desses fatores e a duração do procedimento anestésico-cirúrgico evidenciam o maior ou menor risco de desenvolvimento de LP, que podem ser observadas após o término do procedimento e se potencializar rapidamente. Os locais mais comuns para desenvolvimento de LP por posicionamento cirúrgico são: região sacral, calcâneos, região mentoniana e trocânteres ${ }^{5,6}$.

Para posicionar adequadamente e de forma segura o paciente cirúrgico na mesa operatória é imprescindível que o enfermeiro do centro cirúrgico (CC) tenha conhecimento das alterações anatômicas e fisiológicas decorrentes do posicionamento cirúrgico no organismo, bem como dos equipamentos e dispositivos disponíveis para auxiliar na execução do procedimento e, portanto, reduzir a taxa de LP em pacientes submetidos a procedimentos anestésico-cirúrgicos ${ }^{7,8}$.
Desse modo, é essencial que todos os membros da equipe cirúrgica compreendam o reconhecimento dos fatores de risco relacionados à ocorrência de LP no perioperatório e que apliquem efetivamente protocolos de prevenção de LP no CC, direcionando, assim, suas condutas assistenciais perioperatórias conforme as reais necessidades do paciente cirúrgico ${ }^{9-11}$.

Diante da etiologia multifatorial das lesões cirúrgicas, todos os pacientes que serão submetidos a alguma intervenção cirúrgica devem ser sistematicamente avaliados durante o período perioperatório, a fim de que riscos sejam identificados e de que seja preparado um plano de cuidados individualizado, que garanta uma assistência perioperatória de qualidade ${ }^{4-6}$.

\section{OBJETIVO}

Avaliar a ocorrência de LP no período intraoperatório decorrentes de procedimentos cirúrgicos realizados em um hospital universitário de grande porte.

\section{MÉTODO}

Trata-se de um estudo de corte transversal, de caráter descritivo, com abordagem quantitativa, desenvolvido na unidade de CC e nas enfermarias cirúrgicas de um hospital universitário de grande porte do Nordeste do Brasil. O CC é composto de dez salas operatórias, e os procedimentos cirúrgicos ocorrem de acordo com o mapa de distribuição das salas para as diversas especialidades. São realizadas, em média, entre 350 e 400 cirurgias por mês no serviço, apenas de caráter eletivo, incluindo 14 especialidades cirúrgicas e cirurgias de alta complexidade como cirurgias cardíacas, torácicas, neurológicas, oncológicas e transplantes.

A população foi constituída de pacientes submetidos à cirurgia no período de coleta, incluídos os maiores de 18 anos e que não estavam sendo incluídos em cirurgias de urgência ou reoperações. Os critérios de exclusão foram pacientes com déficit cognitivo, alta hospitalar em tempo inferior a 24 horas do término da cirurgia e encaminhamento para a unidade de terapia intensiva após procedimento anestésico-cirúrgico.

A amostra foi obtida por conveniência. Para estimar o tamanho da amostra, utilizou-se o cálculo para estimativa de médias, considerando-se como desfecho principal a variável quantitativa escore de risco, medida pela Escala de Avaliação de Risco para o Desenvolvimento de Lesões Decorrentes do Posicionamento Cirúrgico (ELPO), desfecho principal 
do estudo. Foi considerado um desvio padrão (DP) de 3,84; extraído do estudo de elaboração da escala de $\mathrm{ELPO}^{7}$, um erro máximo estimado em 0,5 ponto e nível de significância para os testes estatísticos de $5 \%$. Sendo assim, para a média mensal de 370 cirurgias / mês no ano anterior e o período de coleta de três meses, a amostra calculada foi de 189 pacientes. Foram abordados, contudo, 227 pacientes, dos quais 154 preencheram os critérios de inclusão. Houve perda de amostragem de 73 pacientes, dos quais 43 receberam alta hospitalar antes das 24 horas, 12 cirurgias foram canceladas/suspensas, sete pacientes se encontravam impossibilitados de comunicação verbal por conta da intubação orotraqueal, seis possuíam algum tipo de limitação cognitiva ou estavam em uso de sedativos e cinco não aceitaram participar do estudo.

A coleta de dados foi executada em dois momentos. No período pré-operatório imediato, os pacientes foram identificados na visita pré-operatória, convidados a participar da pesquisa e orientados sobre o protocolo do estudo. Mediante o aceite do paciente em participar da pesquisa, foi entregue o Termo de Consentimento Livre e Esclarecido, com todas as informações e esclarecimentos sobre o estudo. Os dados sociodemográficos, clínicos e avaliação das condições da pele do paciente internado foram coletados na enfermaria cirúrgica de origem do paciente, utilizando-se instrumento específico para registro das observações.

No primeiro dia de pós-operatório foram realizadas as segundas avaliações em uma nova visita ao paciente na enfermaria cirúrgica de origem, a partir de 24 até 48 horas após o procedimento anestésico-cirúrgico, com a finalidade de verificar se ocorreu o desenvolvimento de LP, por meio da inspeção das condições da pele, completando assim o preenchimento do instrumento da coleta. Os dados cirúrgicos (procedimento realizado, posicionamento cirúrgico, tempo cirúrgico, tipo de anestesia, classificação pela American Society of Anesthesiologists ASA, localização da placa de bisturi, complicações, uso de hemoderivados, preparo da pele, uso na pele de fitas adesivas e tricotomia) foram obtidos no prontuário do paciente, na ficha de intraoperatório (preenchida pelo anestesiologista), na descrição do ato cirúrgico (preenchida pelo cirurgião) e na ficha de enfermagem perioperatória (preenchida pela equipe de enfermagem). Os dados pós-operatórios não foram coletados na sala de recuperação pós-anestésica pelas dificuldades de comunicação e mobilização do paciente.

A ELPO foi utilizada para avaliação do escore de risco e para avaliação dos fatores associados à lesão. A ELPO foi criada e validada no Brasil, no ano de 2013, com objetivo de avaliar o risco para o desenvolvimento de lesões tegumentares (lesões por pressão), lesões neurológicas (lesões em nervos) e dor não relacionada à incisão cirúrgica ${ }^{7}$.

A escala contém sete itens: tipo de posicionamento cirúrgico, tempo de cirurgia, tipo de anestesia, superfície de suporte, posição dos membros, comorbidades e idade do paciente. O escore 20 consiste no ponto de corte para diferenciar os pacientes classificados pela ELPO, ou seja, o paciente com escore até 19 pontos é classificado com risco menor para o desenvolvimento de lesões decorrentes do posicionamento cirúrgico, e aquele com escore 20 ou mais é classificado com risco maior. Essa classificação permite estabelecer quais pacientes possuem maior risco e, consequentemente, quais a equipe perioperatória deverá ter mais cautela durante a execução do posicionamento, para prevenir complicações associadas ao procedimento cirúrgico ${ }^{7}$.

Para avaliar as LP identificadas no pós-operatório, adotou-se a classificação preconizada pela National Pressure Ulcer Advisory Panel (NPUAP), adaptada culturalmente para o Brasil para as LP, mas foram incluídas outras lesões, como a abrasão ${ }^{12}$.

Os dados foram tabulados no programa Microsoft Excel e analisados pelo software livre Statistical Package for the Social Sciences (SPSS). Foram utilizados recursos de estatística descritiva (frequências absoluta e relativa, médias e DP) e para análise dos dados foi usado o teste $t$ de Student para comparar médias de amostras independentes. Dados referentes às variáveis da ELPO, de acordo com presença ou ausência de LP no período intraoperatório, foram analisados pelo teste $t$ de Student.

A pesquisa foi elaborada pautada nos preceitos éticos da Resolução ${ }^{\circ}$ 466 / 2012, do Conselho Nacional de Saúde, sendo iniciada após aprovação do Comitê de Ética em Pesquisa da instituição, sob parecer $n^{\circ}$ 2.045.355.

\section{RESULTADOS}

Dos pacientes que participaram do estudo $(\mathrm{n}=154)$, a maioria ( $\mathrm{n}=102 / 66,2 \%)$ era do sexo feminino; com média de idade de $51,8 \pm 15,4$ anos, $37 \%(n=57)$ idosos; $21,4 \%(n=33)$ aposentados. Dos 154 pacientes, $23,3 \%(n=36)$ eram da capital, $37 \%$ $(\mathrm{n}=57)$ moravam na região metropolitana, $33,7 \%(\mathrm{n}=52)$ no interior e $5,8 \%(n=9)$ eram de outro estado do Nordeste. A maior parte já havia sido submetida a uma cirurgia anteriormente $(\mathrm{n}=108 / 70,1 \%)$.

Dos pacientes avaliados, 53,9\% $(\mathrm{n}=83)$ apresentaram como comorbidade a hipertensão arterial sistêmica, seguida por diabetes mellitus ( $\mathrm{n}=26 / 16,8 \%)$, etilismo $(\mathrm{n}=28 / 18,2 \%)$ e tabagismo $(n=27 / 17,5 \%)$. Quanto ao índice de massa 
corpórea (IMC), a média foi de $26,7 \mathrm{~kg} / \mathrm{m}^{2}$, variando entre 16,7 e $51 \mathrm{~kg} / \mathrm{m}^{2}$, e $21,1 \%(\mathrm{n}=31)$ dos pacientes apresentaram padrões de obesidade.

Em relação à limitação física, 14 (9\%) pacientes precisavam de ajuda para deambular, $8(5,1 \%)$ relataram possuir algum tipo de limitação de movimento devido ao sobrepeso, $8(5,1 \%)$ pacientes eram acamados e $1(0,6 \%)$ era portador de paraplegia.

A avaliação das condições da pele no período pré-operatório revelou que 147 (95,5\%) pacientes não apresentavam lesões cutâneas nesse período, entretanto 7 (4,5\%) foram admitidos no CC com algum tipo de LP, entre elas: pé diabético/lesão vascular em pé/perna (37,5\%), escoriações (12,5\%), doença dermatológica do tipo psoríase (12,5\%), queloide (25\%) e infecção de partes moles (12,5\%).

A especialidade cirúrgica mais frequente foi a cirurgia geral $(n=47 / 30,5 \%)$, seguida pela ginecologia $(n=44 / 28,5 \%)$ e urologia $(n=34 / 22 \%)$. As cirurgias mais realizadas foram laparotomia exploratória e histerectomia total ampliada, ambas com 19 pacientes $(12,3 \%)$ cada, seguidas pela colecistectomia videolaparoscópica ( $\mathrm{n}=11 / 7,14 \%)$.

Verificou-se, de acordo com a avaliação da ELPO, que $72(46,7 \%)$ procedimentos anestésico-cirúrgicos realizados tiveram duração entre 2 até 4 horas e $7(4,6 \%)$ procedimentos duraram mais do que 6 horas (Tabela 1 ).

Observou-se que $39,7 \%(\mathrm{n}=61)$ dos procedimentos utilizaram anestesia regional e $68,2 \%(n=105)$ adotaram a posição cirúrgica de decúbito dorsal; e na totalidade dos procedimentos ( $\mathrm{n}=154 / 100 \%)$ foi utilizada a mesa de operação padrão (MOP) com colchão de espuma e coxins feitos de campo de algodão como superfícies de suporte para os pacientes (Tabela 1). Em relação à posição dos membros (superiores e inferiores), verificou-se que em $110(71,4 \%)$ procedimentos avaliados a abertura dos membros superiores foi menor do que $90^{\circ}$ (Tabela 1 ).

Dos procedimentos cirúrgicos, observou-se que $88,3 \%$ $(\mathrm{n}=136)$ utilizaram o eletrocautério (bisturi elétrico), sendo a placa posicionada principalmente na panturrilha $(n=65 / 42,2 \%)$, no vasto lateral da coxa $(\mathrm{n}=51 / 33,1 \%)$ e no tórax posterior $(\mathrm{n}=15 / 9,7 \%)$.

Com relação às intercorrências no intraoperatório, identificou-se a ocorrência de apenas $8(5,1 \%)$ casos, sendo: hipotensão, hipertensão, sangramento intenso, hipoxemia, hipoglicemia, hiperglicemia e oligúria.

Em mais da metade dos pacientes ( $\mathrm{n}=92 / 59,7 \%)$ não foi realizada tricotomia; $43(27,9 \%)$ afirmaram ter realizado a tricotomia mais de 2 horas antes do procedimento anestésico-cirúrgico e $19(12,4 \%)$ realizaram a tricotomia em até 2 horas antes da cirurgia.

Os resultados do escore da ELPO no período intraopera-

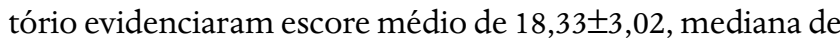
19 pontos, com escore mínimo de 11 e máximo de 27 pontos. A maioria dos pacientes avaliados $(n=103 / 66,9 \%)$ apresentou baixo risco de desenvolver LP no período intraoperatório, segundo a ELPO ( $\leq 20$ pontos). Apenas $7(4,5 \%)$ pacientes apresentaram LP ao término do procedimento anestésico-cirúrgico, sendo que $4(57,1 \%)$ apresentaram mais do que uma lesão, totalizando, assim, 11 registros de lesões.

Tabela 1. Distribuição dos pacientes submetidos a cirurgias eletivas ( $n=154$ ), segundo variáveis presentes na Escala de Avaliação de Risco para o Desenvolvimento de Lesões Decorrentes do Posicionamento Cirúrgico.

\begin{tabular}{|l|l|l|}
\hline Variáveis & n & $\%$
\end{tabular}

Tempo de duração da cirurgia (horas)

Até 2

Acima de 2 até 4 $59 \quad 38,3$

Acima de 4 até 6 72 46,7

Acima de 6 $16 \quad 10,4$

Tipo de anestesia

\begin{tabular}{|l|c|c|}
\hline Local & 1 & 0,6 \\
\hline Sedação & 1 & 0,6 \\
\hline Regional & 61 & 39,7 \\
\hline Geral & 60 & 39,0 \\
\hline Geral + regional & 31 & 20,1 \\
\hline
\end{tabular}

Tipo de posição cirúrgica

Supina $105 \quad 68,2$

Lateral $13 \quad 8,4$

Trendelemburg $0 \quad 0,0$

Prona

32,0

Litotômica $33 \quad 21,4$

Tipo de suporte

Colchão de espuma + coxins de campos de algodão

Posicionamento cirúrgico dos membros

\begin{tabular}{l|ll} 
Posição anatômica & 1 & 0,65
\end{tabular}

\begin{tabular}{ll|l|l} 
Abertura dos membros superiores $<90^{\circ}$ & 110 & 71,4
\end{tabular}

Elevação dos joelhos $<90^{\circ}$ e abertura dos \begin{tabular}{ll|l|l|} 
membros inferiores $<90^{\circ}$ ou pescoço sem & 40 & 26,0
\end{tabular} alinhamento mento-esternal

Elevação dos joelhos $>90^{\circ}$ ou abertura dos membros inferiores $>90^{\circ}$

32,0


A Tabela 2 apresenta os casos de LP decorrentes do intraoperatório registrados.

A principal especialidade a apresentar LP foi a cirurgia ortopédica $(n=4 / 57,1 \%)$. O posicionamento mais adotado para os pacientes com LP foi o decúbito dorsal ( $n=3 / 42,8 \%$ ), seguido pela posição de decúbito lateral esquerdo e decúbito ventral, ambas com $2(28,6 \%)$ pacientes cada. O tipo prevalente de procedimento anestésico foi a anestesia geral e a forma combinada (geral + regional), cada um com 3 pacientes $(42,8 \%)$, e apenas $1(14,3 \%)$ paciente foi submetido à anestesia regional. Em relação à classificação ASA, $4(57,1 \%)$ foram classificados como ASA II, 2 (28,6\%) ASA III e 1 (14,3\%) ASA I. Na Tabela 3 estão listadas as caracterizações de LP e suas frequências, encontradas na amostra deste estudo.
No primeiro dia de pós-operatório, dos 7 pacientes que apresentaram LP, 5 (71,4\%) apresentaram dor não relacionada ao procedimento cirúrgico e sim por causa das LP, ocasionadas no período intraoperatório. A intensidade da dor no local da lesão foi avaliada de acordo com o escore do relato do paciente e variou de 1 a 8 , sendo que houve frequência maior do escore 1 , relatada por 2 pacientes, seguido pelos escores de 3 a 8, relatados por 1 paciente.

Em relação ao escore da ELPO, variou de 17 a 26 pontos, evidenciando escore médio de $21,71 \pm 2,69$ nos pacientes que apresentaram LP no período intraoperatório. Salienta-se que a maior parte dos pacientes avaliados que apresentaram $L P(n=6 / 85,71 \%)$ foi classificada pela ELPO como alto risco (Tabela 3).

Tabela 2. Caracterização dos pacientes com lesões de pele decorrentes do período intraoperatório.

\begin{tabular}{|l|l|c|c|c|c|}
\hline Caso & Sexo & Idade & IMC & Comorbidades & Limitação física \\
\hline 1 & Feminino & 57 & 44 & DM/HAS/CA/obesidade & Nenhuma \\
\hline 2 & Masculino & 37 & 17,9 & CA/desnutrição & Nenhuma \\
\hline 3 & Feminino & 67 & 24,5 & HAS & Deambula com ajuda \\
\hline 4 & Feminino & 60 & 20,8 & Sem comorbidades & Nenhuma \\
\hline 5 & Masculino & 43 & 22,2 & Sem comorbidades & Acamado \\
\hline 7 & Masculino & 52 & 25,4 & Sem comorbidades & Nenhuma \\
\hline
\end{tabular}

IMC: índice de massa corpórea; DM: diabetes mellitus; HAS: hipertensão arterial sistêmica; CA: câncer; DV: doença vascular.

Tabela 3. Caracterização clínica, tipo e localização das lesões, pontuação e classificação da Escala de Avaliação de Risco para o Desenvolvimento de Lesões Decorrentes do Posicionamento Cirúrgico dos pacientes submetidos à cirurgia que apresentaram lesões de pele no período intraoperatório $(n=7)$.

\begin{tabular}{|c|c|c|c|c|c|c|c|c|c|c|}
\hline Caso & $\begin{array}{l}\text { Cirurgia } \\
\text { realizada }\end{array}$ & $\begin{array}{c}\text { Local da placa } \\
\text { de bisturi }\end{array}$ & $\begin{array}{l}\text { Tipo de } \\
\text { posição }\end{array}$ & $\begin{array}{l}\text { Duração } \\
\text { (em horas) }\end{array}$ & $\begin{array}{c}\text { Tipo de } \\
\text { anestesia }\end{array}$ & ASA & $\begin{array}{l}\text { Tipo de lesão } \\
\text { de pele }\end{array}$ & $\begin{array}{c}\text { Local da } \\
\text { lesão de pele }\end{array}$ & Dor* & ELPO \\
\hline 1 & Colectomia & Coxa & Dorsal & 4 & Combinada & II & $\begin{array}{l}\text { Região supra } \\
\text { púbica/perianal }\end{array}$ & Abrasão/LP II & 04 & 21 \\
\hline 2 & Colecistectomia & Panturrilha & Dorsal & 9 & Combinada & ॥ & Glúteo E/MID & LP II/abrasão & 05 & 22 \\
\hline 3 & $\begin{array}{l}\text { Artroplastia } \\
\text { de joelho }\end{array}$ & Dorso & Dorsal & 3 & Raqui & II & Sacro coccígea & LPI & 01 & 18 \\
\hline 4 & Nefrectomia & Coxa & Lateral & 4,3 & Combinada & II & Glúteo D & LPI & 01 & 21 \\
\hline 5 & $\begin{array}{l}\text { Artrodese } \\
\text { de quadril }\end{array}$ & Glúteo & Lateral & 8 & Geral & III & Dorso/tórax & $\begin{array}{l}\text { Abrasão/ } \\
\text { abrasão }\end{array}$ & 03 & 21 \\
\hline 6 & $\begin{array}{c}\text { Descompressão } \\
\text { lombar }\end{array}$ & Coxa & Ventral & 5,5 & Geral & I & $\begin{array}{c}\text { Região } \\
\text { zigomática }\end{array}$ & LPI & 07 & 22 \\
\hline 7 & $\begin{array}{l}\text { Revisão de } \\
\text { artrodese cervical }\end{array}$ & Coxa & Ventral & 8 & Geral & II I & Tórax bilateral & LP I/LP ॥ & 08 & 27 \\
\hline
\end{tabular}


Os pacientes que apresentaram LP no período intraoperatório obtiveram média de $21,7 \pm 2,7$ na escala de ELPO. Em contrapartida, os pacientes que não apresentaram LP apresentaram média de 18,21士3,0.

A Tabela 4 demonstra que os pacientes com lesões apresentaram resultados piores (escores mais elevados) nos domínios tempo de cirurgia, tipo de anestesia e comorbidades, não sendo significativos a diferença apenas para idade, a posição dos membros nem o tipo de posição cirúrgica. A superfície cirúrgica foi sempre a mesma nos dois grupos, visto que no hospital há apenas um tipo de colchão para mesa operatória e não coxins de gel, nem de viscoelástico, sendo usados lençóis com coxins para posicionamento.

\section{DISCUSSÃO}

Os cuidados de enfermagem dedicados ao paciente no período intraoperatório refletirão no pós-operatório, pois muitas LP têm seu princípio na sala de operação, intensificando-se na fase do pós-operatório. Os pacientes cirúrgicos são os primeiros candidatos à isquemia tissular, por conta da diminuição do fluxo capilar, por tempo prolongado de imobilidade e pressão no procedimento cirúrgico, conferindo ao paciente maior intolerância à pressão. Sendo assim, é indispensável a manutenção da integridade cutânea de cada paciente, necessitando que o enfermeiro se aproprie do conhecimento técnico e científico ${ }^{9-13}$.

Estudos descrevem que existe associação do desenvolvimento de LP decorrentes do posicionamento cirúrgico com os vários fatores de risco relacionados à ocorrência dessas lesões, destacando-se principalmente os fatores intrínsecos como idade, peso corporal, estado nutricional, doenças crônicas (diabetes mellitus, neuropatias, hipertensão arterial e anemia) e os fatores extrínsecos como tipo e tempo de cirurgia, tipo de anestesia, posições cirúrgicas adotadas e recursos e medidas utilizadas para proteção ${ }^{6-14}$.

A prevalência do sexo feminino (66,2\%) nos pacientes submetidos a cirurgias eletivas permaneceu equivalente, quando comparada a outros estudos ${ }^{7-15}$. Quanto à faixa etária, grande parte dos pacientes era adulta (63\%). Pessoas idosas possuem maior risco de complicações perioperatórias, até mesmo maior risco de desenvolver LP no período intraoperatório, quando comparadas a pessoas adultas, por possuírem menor espessura da pele, diminuição da massa muscular e da gordura subcutânea sobre as proeminências ósseas, deixando-as mais suscetíveis à pressão e, consequentemente, ao surgimento de danos teciduais ${ }^{14-16}$.

Além da idade, a presença de comorbidades como diabetes mellitus, vasculopatias, neuropatias e hipertensão arterial é fator de risco para a ocorrência de lesões perioperatórias por posicionamento, uma vez que tais patologias comprometem a perfusão tissular ${ }^{6-8,14-17}$. Hipertensão e diabetes, ambas entidades encontradas em proporção significativa na amostra, são as comorbidades responsáveis por aumentar o risco de $\mathrm{LP}^{8-18}$.

Quanto ao estado nutricional, o baixo peso e o sobrepeso, indicados pelo IMC, são também fatores de risco associados ao aparecimento de lesões ${ }^{17}$. Os pacientes com obesidade e sobrepeso apresentam pouca vascularização do tecido gorduroso, levando a procedimentos cirúrgicos mais demorados, à maior facilidade de trauma do tecido operado e à dificuldade de realizar hemostasia adequada. Por outro lado, o baixo peso acarreta exposição acentuada de proeminências ósseas, deixando os pacientes mais suscetíveis à ocorrência de LP decorrentes do posicionamento cirúrgico ${ }^{4-14}$.

Tabela 4. Dados referentes às variáveis da Escala de Avaliação de Risco para o Desenvolvimento de Lesões Decorrentes do Posicionamento Cirúrgico, de acordo com presença ou ausência de lesão de pele no período intraoperatório.

\begin{tabular}{|c|c|c|c|}
\hline ELPO & $\begin{array}{l}\text { Sem lesão de pele }(n=147) \\
\text { média } \pm D P\end{array}$ & $\begin{array}{c}\text { Com lesão de pele }(n=7) \\
\text { média } \pm \text { DP }\end{array}$ & $\mathbf{p}^{*}$ \\
\hline \multicolumn{4}{|l|}{ Variável ELPO } \\
\hline Tipo de posição cirúrgica & $2,02 \pm 1,66$ & $1,63 \pm 1,41$ & 0,465 \\
\hline Tempo de cirurgia & $2,69 \pm 0,86$ & $4,75 \pm 1,17$ & 0,001 \\
\hline Tipo de anestesia & $3,73 \pm 0,78$ & $4,63 \pm 0,52$ & 0,002 \\
\hline Superfície de suporte & $4 \pm 0,00$ & $4 \pm 0,00$ & ** \\
\hline Posição dos membros & $2,29 \pm 0,51$ & $2,25 \pm 0,46$ & 0,52 \\
\hline Comorbidades & $2,25 \pm 1,35$ & $3,37 \pm 1,06$ & 0,02 \\
\hline Idade & $2,25 \pm 1,31$ & $2,12 \pm 0,83$ & 0,649 \\
\hline Escore ELPO & $18,21 \pm 3,05$ & $21,75 \pm 2,69$ & 0,06 \\
\hline
\end{tabular}


Os dispositivos utilizados para diminuir as interfaces de pressão durante o procedimento anestésico-cirúrgico podem ser classificados como estáticos e dinâmicos. Os dispositivos estáticos são os colchões de espuma e gel, as coberturas de colchão de gel e polímero de viscoelástico seco, colchões de ar ou fluido e almofadas de espuma e gel. Já os dispositivos dinâmicos estudados são os colchões de ar micropulsantes ${ }^{19}$. O serviço ainda não dispõe desses recursos, tendo apenas o colchão tradicional e improvisando coxins com os campos de tecido de algodão.

Um achado importante e que merece ser ressaltado é relacionado à ausência de lesões (queimaduras) por placa de bisturi elétrico (eletrocautério) nos pacientes avaliados. Sabe-se que o risco de queimaduras está associado principalmente à colocação incorreta da placa de eletrocautério e a instalações elétricas inadequadas ${ }^{9}$. Contudo, no serviço são utilizadas apenas placas descartáveis, com propriedade adesiva, e os bisturis possuem sistema de segurança, que impede seu uso se a placa não tiver sido conectada ou se estiver fora de uso ${ }^{19}$.

O tempo cirúrgico evidenciado nesta pesquisa, com duração maior do que 2 horas, também é citado como um dos fatores risco para ocorrência de lesões, visto que períodos longos de imobilização e de exposição à pressão causam anóxia, necrose tecidual e, consequentemente, $\mathrm{LP}^{6-9}$.

A imobilidade prolongada do paciente sobre a mesa cirúrgica diminui o volume do fluxo de sangue capilar pulmonar, limitando assim a expansão pulmonar, por conta da pressão da posição sobre as costelas ou da capacidade de o diafragma forçar o conteúdo abdominal para baixo ${ }^{17}$.

Outro fator de risco no intraoperatório é o tipo de anestesia, que influencia no grau de depressão do sistema nervoso, em que ocorre vasodilatação periférica, resultando em hipotensão e diminuição do retorno venoso, depressão dos receptores da dor, fazendo com que os mecanismos de defesa do paciente não ofereçam mais proteção contra pressão, deixando-os suscetíveis a lesões perioperatórias decorrentes do posicionamento ${ }^{17-20}$.

A posição supina foi a posição cirúrgica que obteve frequência maior (68,2\%), o que corrobora o estudo de Lopes, no qual, dos 115 procedimentos cirúrgicos, 83 (72,2\%) foram realizados na posição supina ou prona ${ }^{16}$. Em outra pesquisa, realizada com pacientes cirúrgicos em um hospital do Triângulo Mineiro, Minas Gerais, a posição supina foi adotada em $50 \%$ dos pacientes ${ }^{9}$.

Em outro estudo nacional, em 251 (90,3\%) procedimentos anestésico-cirúrgicos foi utilizado o colchão da mesa cirúrgica de espuma (convencional) e os coxins improvisados com campos de algodão. Outro estudo expôs que o colchão da mesa cirúrgica de espuma e os coxins feitos de campos de algodão foram utilizados em $69(60 \%)$ cirurgias $^{16}$.
As superfícies de suporte são dispositivos especializados, sobreposições, colchões ou sistemas integrados fabricados para a redistribuição da pressão, controle do cisalhamento ou forças de fricção sobre o tecido, manutenção do microclima ou outras funções terapêuticas e devem ser escolhidos de acordo com as necessidades particulares do paciente e do tipo de cirurgia. Têm como objetivo evitar atritos, prevenindo LP, compressão ou estiramentos neuromusculares, contato com o metal da mesa, que pode ocasionar queimaduras (devido ao uso do bisturi elétrico) e outros danos ${ }^{21,22}$. Todavia, as superfícies de suporte são pouco utilizadas nos pacientes cirúrgicos, uma vez que as questões políticas, econômicas e sociais enfrentadas pela saúde no país não permitem que muitos serviços públicos disponham desses tipos de recursos tecnológicos, interferindo diretamente na prevenção de lesões ${ }^{9-20}$.

As cirurgias foram realizadas no mesmo tipo de mesa operatória padrão, com os mesmos coxins improvisados com campos de algodão e utilizou-se o aparelho de bisturi elétrico (eletrocautério) sempre da mesma marca e modelo, com o mesmo tipo de placa descartável. Sendo assim, não houve diferença entre o material utilizado nas cirurgias. Também não houve diferença no preparo da pele, seguindo em todas as cirurgias o mesmo protocolo institucional.

Outros estudos apresentaram discordância, pois as incidências de LP foram bastante elevadas, como apontado nos estudos realizados no estado de São Paulo, que evidenciaram incidências de lesões perioperatórias por posicionamento de 20,6 e $20,9 \%$, respectivamente ${ }^{6,18}$. No estado de Minas Gerais, pesquisas realizadas identificaram taxas de incidência em que 21,7 e $74 \%$ dos pacientes apresentaram LP ao término do procedimento cirúrgico ${ }^{7,9}$. Conforme destacado, a incidência de lesões perioperatórias decorrentes do posicionamento permanece alta por causa da ausência de medidas preventivas para evitar tais lesões, sendo a não adesão ou verificação de normas e / ou protocolos de diretrizes clínicas o principal fator contribuinte ${ }^{3-20}$.

Em uma pesquisa com pacientes submetidos a cirurgias urológicas robóticas, os autores apontam incidência zero, detectada no período pós-operatório imediato, sendo essa taxa de incidência justificada pela efetividade do protocolo institucional de prevenção de LP no CC ${ }^{11}$. Tal resultado demonstra que os treinamentos com simulação da equipe interdisciplinar e multiprofissional, bem como a implementação de estratégias de prevenção e protocolos, são essenciais para garantir a efetividade da segurança do paciente no ambiente cirúrgico.

Ao analisar o escore da dor nos pacientes que apresentaram LP após o procedimento anestésico-cirúrgico, todos relataram sentir dor não relacionada à incisão cirúrgica e sim ao local da 
LP ocasionada no período intraoperatório. Pacientes com escore mais alto na ELPO têm mais probabilidade de apresentar dor no pós-operatório, em decorrência do posicionamento cirúrgico ${ }^{16}$.

Os estudos sobre lesão perioperatória no paciente cirúrgico indicam que a lesão de estágio I é a mais frequente, sendo esse dado corroborado pela presente pesquisa, na qual $34 \%$ das lesões foram classificadas em LP estágio $I^{6-8,13-18}$. Além disso, pesquisas citam que a maioria dessas lesões evolui para resolução, indicando os cuidados no pós-operatório como decisivos para a melhora da lesão $0^{13-18}$.

A despeito de outros trabalhos que encontraram que as regiões sacral e calcânea foram as mais acometidas no intraoperatório dos pacientes investigados, na nossa amostra os locais principais foram tórax e região glútea ${ }^{6-9,15}$.

Ao analisarmos as médias do escore da ELPO nos pacientes que não apresentaram LP versus os que apresentaram, houve diferença de quase 4 pontos entre os grupos, próxima ao estudo de Lopes, que apresentou diferença de quase 5 pontos entre as médias da ELPO nos dois grupos, sendo estatisticamente significante $(\mathrm{p}<0,001)^{16}$. Embora não tenha sido significativa essa diferença $(p=0,06)$, constatou-se que os pacientes com lesão apresentaram maiores escores na ELPO e, além disso, $85,7 \%$ dos pacientes com lesão apresentaram escore ELPO maior do que 20 pontos, corroborando a inferência de que escores elevados da ELPO têm associação com maior incidência de LP perioperatória.

Em relação ao escore ELPO e à ocorrência de lesões, Lopes et al. identificaram a associação do escore da ELPO com o desenvolvimento de lesão perioperatória por posicionamento, evidenciando que cada ponto a mais em que o sujeito é classificado na escala aumenta $44 \%$ a probabilidade de desenvolver $\mathrm{LP}^{7}$. No presente estudo, $66,9 \%$ dos pacientes obtiveram classificação ELPO em baixo risco de desenvolver lesões perioperatórias, corroborando o estudo de Lopes et al., no qual a maioria dos pacientes $(53,2 \%)$ obteve escore ELPO $\geq 19$ pontos, ou seja, classificados em baixo risco para desenvolver LP decorrente do posicionamento cirúrgico ${ }^{7}$. Em outra população, 56,5\% dos pacientes apresentaram maior risco de desenvolver lesões perioperatórias, segundo a ELPO ${ }^{15}$. Ao analisarmos apenas os sete pacientes que apresentaram LP no intraoperatório, em relação à classificação de risco da ELPO, seis deles obtiveram alto risco para o desenvolvimento desse tipo de lesão.
Por meio da aplicação do teste $t$ de Student para comparação entre as médias dos escores das variáveis da ELPO, constou-se significância nos domínios tempo de cirurgia, tipo de anestesia e comorbidades. Isso remete-se às questões diretamente relacionadas aos fatores de risco para ocorrência de lesões perioperatórias, tanto intrínsecos quanto extrínsecos, evidenciados nesta pesquisa.

Desse modo, a identificação do risco e da ocorrência de lesões, aliada aos fatores associados, pode gerar evidências para o desenvolvimento de estratégias e para a implementação de ações efetivas, que auxiliem e direcionem a equipe multidisciplinar na detecção de pacientes com maior risco para o desenvolvimento de lesões, favorecendo a prevenção de complicações na prática clínica ou na solução dessas em tempo adequado.

A equipe de enfermagem deve estar atenta no momento do posicionamento cirúrgico, checar se não há dobras nos lençóis, se foram colocados todos os recursos de proteção adequadamente e se os acessórios de posicionamento disponíveis na instituição estão sendo usados ${ }^{18}$.

Como limitações do estudo, considera-se a não avaliação das lesões neuromusculares, por não ser objetivo do estudo, porém esse tipo de lesão também é considerado lesão perioperatória, sendo uma variável importante para avaliação em estudos futuros. Sugerem-se outras investigações sobre o tema, nas quais a amostragem tenha maior representatividade.

\section{CONCLUSÃO}

Diante dos resultados obtidos, foi possível concluir que, no período intraoperatório, dos 154 pacientes da pesquisa, 7 (4,5\%) apresentaram algum tipo de LP, classificadas em estágio I, estágio II e abrasão. Destes, 6 pacientes apresentaram escore ELPO maior do que 20 pontos. Em relação ao risco para desenvolver LP no período intraoperatório, $66,9 \%$ dos pacientes obtiveram escore ELPO classificado como baixo risco.

Neste estudo, evidenciou-se que as variáveis tempo do procedimento cirúrgico, tipo de anestesia e comorbidades foram os principais fatores de risco, identificados por meio da escala de ELPO, para o surgimento de LP no período intraoperatório nos pacientes submetidos a cirurgias eletivas no hospital universitário em questão.

\section{REFERÊNCIAS}

1. Croteau RJ. Wrong-site surgery in surgical patient safety: essential Information for surgeons in today's environment. Chicago: American College of Surgeons; 2004.
2. Souza LP, Bezerra ALQ, Silva AEBC, Carneiro FS, Paranaguá TTB, Lemos LF. Eventos adversos: instrumento de avaliação do desempenho em Centro Cirúrgico de um hospital universitário. RevEnfermUERJ. 2011;19(1):127-33. 
3. Mendes W, Pavão ALB, Martins M, Moura MLO, Travassos C. Características de eventos adversos evitáveis em hospitais do Rio de Janeiro. Rev Assoc Med Bras. 2013;59(5):421-8. http://dx.doi. org/10.1016/j.ramb.2013.03.002

4. Lopes CMM, Galvão CM. Posicionamento cirúrgico: evidências para o cuidado de enfermagem. Rev Latino-Am Enferm. 2010;18(2):28794. http://dx.doi.org/10.1590/S0104-11692010000200021

5. Spruce L. Back to basics: preventing perioperative pressure injuries. AORN J. 2017;105(1):92-9. https://doi.org/10.1016/j.aorn.2016.10.018

6. Scarlatti KC, Michel JLM, Gamba MA, Gutiérrez MGR. Pressure ulcers in surgery patients: incidence and associated factors. Rev Esc Enferm USP. 2011;45(6):1372-9. http://dx.doi.org/10.1590/ S0080-62342011000600014

7. Lopes CMM, Haas VJ, Dantas RAS, Oliveira CG, Galvão CM. Assessment scale of risk for surgical positioning injuries. Rev Latino-Am Enferm. 2016;24:e2704. http://dx.doi.org/10.1590/1518-8345.0644.2704

8. Saraiva IL, Paula MFC, Carvalho R. Úlcera por pressão no período transoperatório: ocorrência e fatores associados. Rev SOBECC. 2014;19(4):207-13. https://doi.org/10.5327/Z1414-4425201400040006

9. Barbosa MH, Oliva AMB, Sousa Neto AL. Ocorrência de lesões perioperatórias por posicionamento cirúrgico. Rev Cubana Enfer. $2011 ; 27(1): 31-41$

10. Matos FGOA, Piccoli M. Diagnóstico de enfermagem Risco para Lesão Perioperatória por Posicionamento identificado no período transoperatório. Rev Ciênc Cuid Saúde. 2004;3(2):195-201. http:// dx.doi.org/10.4025/cienccuidsaude.v3i2.5426

11. Angelo CS, Pachioni CFM, Joaquim EHG, Silva EAL, Santos GG, Bonfim IM, et al. Efetividade do protocolo prevenção de lesões de pele em cirurgias urológicas robóticas. Rev SOBECC. 2017;22(3):15260. https://doi.org/10.5327/Z1414-4425201700030006

12. National Pressure Ulcer Advisory Panel. Announces a change in terminology from pressure ulcer to pressure injury and updates the stages of pressure injury. Washington, D.C.: National Pressure Ulcer Advisory Panel; 2016.
13. Cruz EA, Soares E. O centro cirúrgico como espaço do cuidado na relação enfermeira/ paciente. Rev SOBECC. 2004;9(2):11-6.

14. Menezes S, Rodrigues R, Tranquada R, Müller S, Gama K, Manso T. Lesões decorrentes do posicionamento para cirurgia: incidência e fatores de risco. Acta Med Port. 2013;26(1):12-6.

15. Peixoto CA. Ocorrência de lesões perioperatórias por posicionamento e fatores associados [dissertação]. Uberaba: Universidade Federal do Triangulo Mineiro; 2017.

16. Lopes CMM. Escala de avaliação de risco para o desenvolvimento de lesões decorrentes do posicionamento cirúrgico: construção e validação [tese]. Ribeirão Preto: Escola de Enfermagem de Ribeirão Preto, Universidade de São Paulo; 2013.

17. Miranda AB, Fogaça AR, Rizzetto M, Lopes LCC. Posicionamento cirúrgico: cuidados de enfermagem no transoperatório. Rev SOBECC. 2016;21(1):52-8. https://doi.org/10.5327/Z1414-4425201600010008

18. Carneiro GA, Leite RCBO. Lesões de pele no intra-operatório de cirurgia cardíaca: incidência e caracterização. Rev Esc Enferm USP. 2011;45(3):611-6. http://dx.doi.org/10.1590/ S0080-62342011000300009

19. Ursi ES, Gavão CM. Prevenção de lesões de pele no perioperatório: revisão integrativa da literatura. Rev Latino-am Enferm. 2006;14(1):12431. http://dx.doi.org/10.1590/S0104-11692006000100017

20. de Oliveira KF, Nascimento KG, Nicolussi AC, Chavaglia SRR, de Araújo CA, Barbosa MH. Support surfaces in the prevention of pressure ulcers in surgical patients: an integrative review. Int J Nurs Pract. 2017;23(4). https://doi.org/10.1111/ijn.12553

21. Grigoleto ARL, Avelar MCQ, Lacerda RA, Mendonça SHF. Complicações decorrentes do posicionamento cirúrgico de clientes idosos submetidos à cirurgia de quadril. Rev Esc Anna Nery. 2011;15(3):531-5. http:// dx.doi.org/10.1590/S1414-81452011000300013

22. Association of periOperative Room Nurses. Proposed recommended practices for safe care through identification of potential hazards in the surgical environment. AORN J. 1995;62(3):455-61. 